\title{
Performance Analysis of Electromagnetic Field Polarizations in MIMO Antenna Systems
}

\author{
Raghav Madan \\ Department of Computer Science and Engineering \\ Maharaja Agrasen Institute of Technology \\ Delhi
}

\begin{abstract}
Due to correlation behavior of the electromagnetic wave components, there is an immense need of deploying impressive polarization techniques in wireless communication systems which could boost the channel capacity. MultipleInput-Multiple-Output (MIMO) antenna system holds a lot of promise to enhance the spectral efficiency. The diversity and signal processing employed with MIMO transforms a pointto-point single channel into multiple parallel channels, hence in effect multiplying the capacity. This paper highlights the importance of electromagnetic field polarization to enhance capacity of the communication channel. In this paper a simulation study to investigate the capacity of orthogonallypolarized MIMO channel under various parameters has been presented. The parameters of study include number of antenna elements, element spacing and eigenvalue analysis of the received signal. We find that the capacity is increased by increasing the number of antenna elements and spacing between them. Furthermore, Simulation result reveals that capacity can be tripled by incorporating MIMO communication techniques to access the entire discernible electric and magnetic polarized fields.
\end{abstract}

\section{Keywords}

Multiple-input Multiple-Output (MIMO), Single-Input SingleOutput (SISO), Channel capacity, Polarization diversity, Electromagnetic waves theory (EMT), Propagation channel, Angular Spectrum

\section{INTRODUCTION}

In recent years, an exponential upsurge in demands in wireless communication networks has been observed. Due to them, an unfaltering expansion in the quantity of users, their ubiquitous demands and capacity exhaustive data applications has addressed both producers and administrators to give sufficient capacity in the systems [1]. Multiple-Input-Multiple-Output (MIMO) system technology has been well-regarded as a critical basis on which to shape the future generations of wireless networks [2]. Unlike traditional communication link like Single-Input Single-Output (SISO) system, MIMO uses multiple antenna elements for transmit and receive purposes. Some of the great benefits of MIMO system are;

1. MIMO technology might make full use of several diversity techniques further than the spatial diversity. These techniques in essence cover frequency diversity, time diversity and polarization diversity respectively [2].

2. MIMO system is likewise part of the IEEE802.11n standard used by wireless router and in addition IEEE802.16 for Mobile WiMax used by cell phone. The LTE standard also incorporates MIMO [2].
3. ITU uses MIMO in the High Speed Downlink Packet Access (HSPDA), part of the UMTS standard [2].

Recently, several researchers have surveyed multiple polarizations for a MIMO antenna system. Andrews et. al [3] contended for a MIMO communication interface that gives six uncorrelated fields with three electric and three magnetic dipoles at the transceiver. An antenna model having ideal polarizations and a multipath enriched environment was presumed, and proved their examination tentatively using three orthogonal electric dipoles [3] [4]. This paper presents the extension of the above study. In this paper, we have examined channel capacity of a wireless system by utilizing MIMO channel having polarization diversity. Polarization diversity [1]-[18] offers a valuable benefit to MIMO system to decrease the signal correlation of local antennas at mobile handsets. Independent signals are transmitted on each horizontal and vertical polarization. Despite of the fact that the channels, at the same frequency, comprise of independent data on the two orthogonally polarized paths. Via combining two corresponding polarizations, this arrangement can inoculate a system from polarization mismatches that would generally cause signal fading. Moreover, such diversity technique has demonstrated significant at wireless communication systems since it is less vulnerable to random orientations of antennas transmitting [5].

\subsection{ORGANIZATION OF THE PAPER}

The paper is presented in following manner; section II presents the basic materials required which concentrates on the basics of electromagnetic wave polarizations and propagation channel respectively. Section III narrates the theoretic MIMO channel modeling that takes into account the characteristics of electromagnetic wave propagation. The simulations and results are discussed in section IV. Finally conclusion is provided in section $\mathrm{V}$.

\section{BASIC FORMULATION}

The proper implementation of polarization diversity for MIMO system requires the understanding of the basic Electromagnetic wave theory (EMT). This section gives emphasis on the material needed to formulate a comprehensive design to enhance channel capacity.

\subsection{Electromagnetics}

The basis for all wireless communications is centered upon considering the radiation and reception of wireless antennas as well as the propagation of electromagnetic fields between these antennas. The concept of radiation, propagation and reception can be enlightened through the use of Maxwell's four foundational equations [6].

$\nabla \times \bar{E}=-\partial \bar{B} / \partial t$ 
$\nabla \times \bar{H}=\partial \bar{D}+\bar{J}$

$\nabla \cdot \bar{D}=\rho$

$\nabla \cdot \bar{B}=0$

Therefore, the equations (1) and (2) becomes after the suppression of time variable $e^{j \omega t}$.

$\nabla \times \bar{E}=-\bar{M}-j \omega \mu \bar{H}$

$\nabla \times \bar{H}=j \omega \varepsilon \bar{E}+\bar{J}$

Where $\bar{E}$ and $\bar{H}$ represents the electric and magnetic fields, $\mu$ and $\varepsilon$ represent the permeability and material permittivity respectively [7].

Using established EMT; consider infinitesimal electric and magnetic current elements radiating into a free space [7][8]. We may orient each of the two current types in the $\mathrm{x}, \mathrm{y}$ and $\mathrm{z}$ directions. Table 1 represents each of these six possible currents. The expressions (1) and (2) together with electric and magnetic currents forms uniquely defined far-field radiation pattern. The electric far-field radiation patterns for all current elements can be expressed in the form;

$\overline{E_{i}}(\theta, \emptyset)=\hat{\theta} E_{i, \theta}+\widehat{\varnothing} E_{i, \emptyset}$

The magnetic currents are not constructed physically but this can be produced using infinitesimal dipoles. Expression (3) indicates both the spatial distribution of fields radiated by the current basis and the strength of the current induced when a field is incident on one of the sensors [7].

Table 1. All Six Electric and magnetic Field Currents [8]

\begin{tabular}{|c|l|l|}
\hline $\begin{array}{c}\text { Current } \\
\text { Orientation }\end{array}$ & Pattern \\
\hline & Electric Current & Magnetic Current \\
\hline$\hat{x}$ & $\begin{array}{c}e_{1} \\
=-\hat{\theta} \cos \theta \operatorname{Cos} \emptyset \\
+\widehat{\emptyset} \sin \emptyset\end{array}$ & $\begin{array}{l}e_{4} \\
=\widehat{\theta} \operatorname{Sin} \emptyset \\
+\widehat{\emptyset} \operatorname{Cos} \theta \operatorname{Cos} \emptyset\end{array}$ \\
\hline$\hat{y}$ & $\begin{array}{l}e_{2} \\
=-\hat{\theta} \cos \theta \operatorname{Sin} \emptyset \\
+\widehat{\emptyset} \cos \emptyset\end{array}$ & $\begin{array}{l}e_{5} \\
=-\hat{\theta} \operatorname{Cos} \emptyset \\
+\widehat{\emptyset} \cos \theta \operatorname{Sin} \emptyset\end{array}$ \\
\hline$\hat{z}$ & $e_{3}=\hat{\theta} \sin \emptyset$ & $e_{6}=-\widehat{\emptyset} \sin \emptyset$ \\
\hline
\end{tabular}

From table 1, it clear that polarization diversity cannot be completely independejnt of angle diversity, since the angular distribution of power is dependent on the polarization of the radiating current [8].

\subsection{Propagation Channel}

In real-time, the propagation channel takes account of no less than two transmission paths. A channel is characterized as the communication path between transmit and receive antennas. The channel represents all conceivable spread ways and in addition the impacts of absorption, spherical spreading, attenuation, reflection losses, Faraday rotation, polarization dependence, delay spread, angular spread, interference and fading [9]. The intricacy of the channel increments as the quantity of accessible propagation ways increments. It likewise grows into more complicated if one or more variables vary with time, for example, the receiver or transmitter position. In normal multipath situations, the dispersion of vast quantities of reflecting, diffracting, refracting and scattering objects become random. Fig. 2 shows a propagation channel with several candidate mechanisms for creating multipath propagation paths [9].

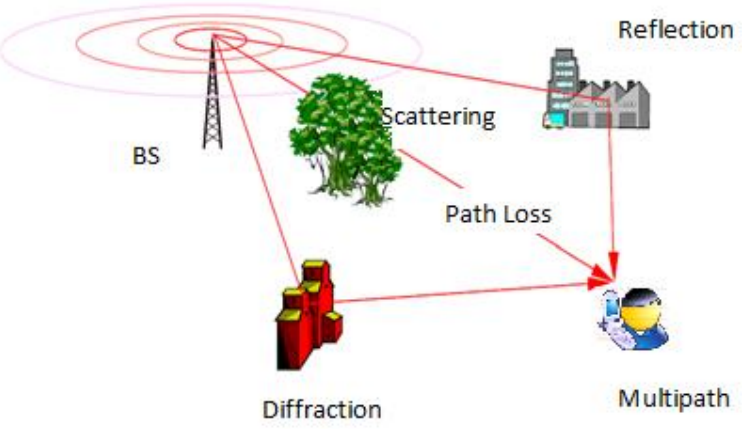

Fig. 1. Multipath Propagation Channel

Therefore, the MIMO antenna systems, when used in multipath propagation channels have tremendous ability to produce striking capacity gains [10]-[12].

\section{MIMO Antenna System 3.1 Diversity in MIMO}

In recent times, the diversity technique was employed to improve the communication framework. Earlier, the hindrance which degraded the wireless performance was the propagation channel. Therefore, in order to enhance the communication system performance the diversity technique was implemented by transmitting the information multiple times. Thus, augmented the likelihood at the receiver end and there might be the possibility of receiving at least one of the signals correctly [13]. In the interim, the polarization diversity can be portraying the utilization of the antenna polarization of the system. Moreover, the polarization mismatch losses and degradation in the information MIMO performance can be evaded by utilizing the polarization diversity [14].

\subsection{MIMO Channel Modelling}

A typical MIMO channel is depicted in Fig. 2 [2]. This shows a MIMO channel where $\mathrm{h} 11=\mathrm{h} 12=\mathrm{h} 21=\mathrm{h} 22$ is the impulse response of the channel. Hence, this impulse response forms the MIMO channel matrix, 'H'. The mathematical equation that described the MIMO system can be expressed as (4), in which ' $x$ ' represents the input signal and ' $y$ ' represent the output signal [2][15].

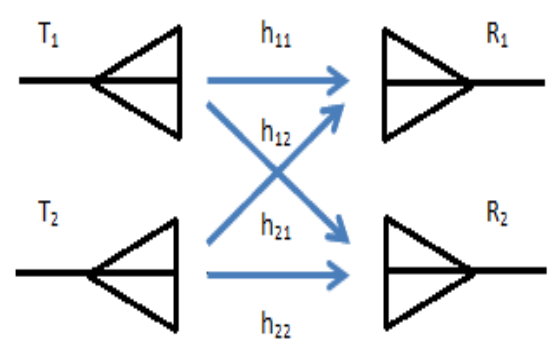

Fig. 2. Traditional MIMO channel 
$y=H x+n$

Where,

$\mathrm{H}=$ MIMO channel matrix, and

$\mathrm{n}=$ Additive White Gaussian Noise (AWGN) in the system

The size of the MIMO channel matrix is depending on the number of transmits and receives antenna. Therefore, (5) shows the MIMO channel matrix, $\mathrm{H}$. N and M represents the number of transmitting and receiving antennas respectively.

$H=\left(\begin{array}{ccc}\rho_{11} & \cdots & \rho_{14} \\ \vdots & \ddots & \vdots \\ \rho_{41} & \cdots & \rho_{y x}\end{array}\right)_{M \times N}$

From (5), correlation coefficient $\rho_{y x}$ is the measure of linear relationship between the output and input signals respectively. The correlation coefficient can be expressed in (6). The covariance between output and input power is written as $\operatorname{cov}_{y x}[15]$.

$\rho_{y x}=\frac{\operatorname{cov}_{y x}}{\sqrt{\sigma_{x x}} \sqrt{\sigma_{y y}}}$

From (6), $\sigma_{x x}$ and $\sigma_{y y}$ represents the variance of input power ' $x$ ' and output power ' $y$ '. The range of the correlation coefficient can be given from-1 to +1 [15]

\subsection{MIMO Channel Capacity with Polarization Diversity}

MIMO channel limit rests vigorously on the factual properties of the channel and antenna element correlations [16]. MIMO channel capacity enumerates the maximum bit rate permitted by channel without error transmission [17]. Channel capacity is defined by;

$C=E\left\{\log _{2}\left[\operatorname{det}\left(I_{n_{R}}+\frac{\rho}{n_{t} N}\left(H H^{H}\right)\right)\right]\right\}$

Where, (.)H is the Hermitian operator known by transposed conjugate matrix, $E\{$.$\} is the expectation, \rho$ is signal to noise ratio $(S N R)$ and $I_{n_{R}}$ is an $n \times m$ identity matrix, and $N$ is the variance of the independent Gaussian noise at each antenna receiver [15]. We utilize the normalized version of the channel which deals with focusing on the effect of channel correlation on the capacity;

$E\left[\|H\|_{F}\right]=\sqrt{n_{T} n_{R}}$

Where, $\|.\|_{F}$ denotes the Frobenius norm [4]. The components of $\mathrm{H}$ are correlated by an amount that relies upon the propagation environs and additionally the polarization of the antenna elements and the spacing between them.

In this paper throughout we have used a method of $\mathrm{H}$ which splits the fading correlation into two independent components as receive and transmit correlation, $H=\Psi_{r}^{1 / 2} \cdot H_{\omega} \cdot \Psi_{t}^{1 / 2}$ [18] [19]. $\Psi_{r}$ and $\Psi_{t}$ are the covariance matrices of $T_{X}$ and $R_{X}$ antennas, and $H_{\omega}$ contains uncorrelated complex Gaussian entries. Assume $i$ and $j$ are the receive antennas, consisting of field patterns $A_{i}$ and $A_{j}$ respectively, are open to the incident field represented by $\bar{E}$. Also assume the phase angles of $E_{\theta}$ and $E_{\varnothing}$ are independent and uniformly distributed in $[0,2 \pi)$, and they are independent for fields arriving from different directions. Thus the $(i, j)^{\text {th }}$ entry of $\Psi_{r}$ is [4];

$\Psi_{r}^{(i, j)}=\frac{1}{\sigma_{i} \sigma_{j}} \oint E\left\{\left(A_{i}(\Omega) \cdot E(\Omega)\right)\left(A_{j}^{*}(\Omega) \cdot E^{*}(\Omega)\right)\right\} d \Omega$

$\Omega$ be the solid angle over $(\theta, \phi)$. Generally $A_{i}$ and $A_{j}$ consists of both amplitude and phase. Assume antennas $i$ and $j$ are collocated with no spatial diversity, it takes after that the phases of $A_{i}$ and $A_{j}$ are similar with respect to angle $\Omega$. From (9), the entries of $\Psi_{r}$ are the weighted correlations between receive antennas, where the weights are the angular spectra of the incident field $\bar{E}$. Keeping in mind the end goal to attain greater channel capacity, $\Psi_{r}$ requires being near to an identity matrix. The correlation coefficient, $\Psi_{r}^{(i, j)}$ for $i \neq j$ can be lessened by diminishing the resemblance in antenna polarizations and/or patterns over the angular space [4].

Similarly, we can also calculate the eigenvalue of the channel. Therefore capacity of the MIMO system is represented as;

$C=\sum_{i=1}^{\min \{N, M\}} \log _{2}\left(1+\frac{E_{S}}{N_{0}} \cdot \frac{1}{N} \cdot \lambda\right)$

Where $\frac{E_{S}}{N_{0}}$ shows the signal to noise ratio, $N$ and $M$ is the transmitting and receiving antenna and $\lambda$ is the eigenvalue. The normalized eigenvalue can be represented by (11).

$\hat{\lambda}_{i}=\frac{\lambda_{i}}{\sum_{j} \lambda_{j}}$

\section{SIMULATION AND RESULTS}

Computer simulation is carried out using MATLAB ${ }^{\circledR}$ 8.0. We suppose that a MIMO channel contains $\mathrm{N}$ antenna elements at the receiving and transmitting ends. The purpose is to illustrate that how various parameters such as number of antenna elements, element spacing, and eigenvector analysis with the increasing angular spectrum would help in increased number of channels.

\subsection{Assumptions Made}

During the simulations, following assumptions were made and several signal properties were exploited.

1. In each component of monochromatic plane-wave, the two orthogonal polarizations are uncorrelated and that plane-wave components separated in angle are also uncorrelated.

2. The incident field has equal power in the two orthogonal polarizations.

3. Additive white Gaussian noise should be zero-mean.

\subsection{Impact of antenna elements on channel capacity}

Using (7), the impact of different number of antenna elements on channel capacity is shown in fig. 3. For this purpose $N_{T}=N_{R}=2,3,4$ are used respectively. The impact of increase in ergodic channel capacity while increasing number of antennas has been observed. Using (12), Shannon's channel capacity is also plotted and the comparison between Shannon's channel capacity with a MIMO channel can be easily observed from fig. 3 .

$C=B \log _{2}\left\{1+\frac{S}{N}\right\}$ 
The result shows that a MIMO antenna effectively gets information from not only Line-Of-Sight (LOS) but also from Non-Line-of-sight(NLOS) environment. Therefore polarization diversity reduces the chances of signal correlation.

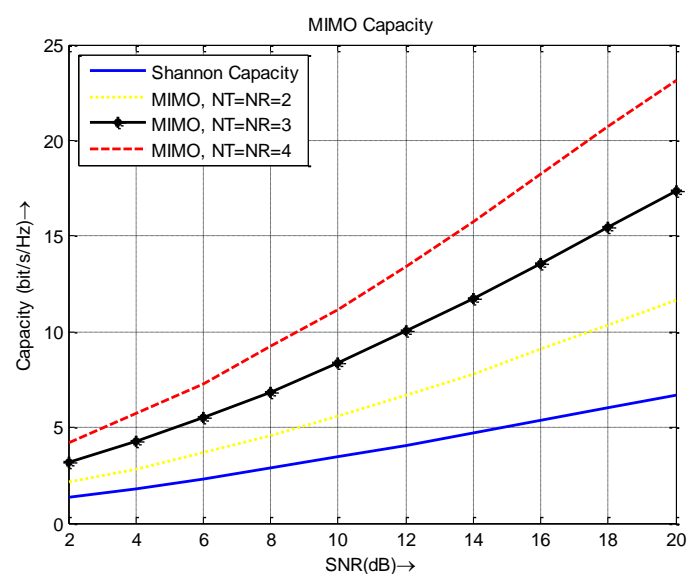

Fig. 3. Number of transmitting and receiving antenna elements as a function of channel capacity (bits/s/Hz) vs. SNR (dB)

\subsection{Impact of Angular spread on channel capacity}

Figure 4 shows the eigenanalysis of the received correlation matrix to access the number of channels available for different incident field characteristics. The result is plotted for three different elevation angles $\Delta \theta=0^{\circ}, 90^{\circ}$ and $180^{\circ}$ whereas each curve represents the sum of eigenvalues. Using (11), the variation of the normalized eigenvalues for different elevation angles is calculated and plotted against angular spread parameter $\Delta \emptyset$. It can be observed for a single propagation path i.e., $\Delta \theta=\Delta \emptyset=0$ only two channels exist corresponding to the two possible polarizations of the incident plane wave. For $\Delta \theta=0^{\circ}$, the eigenvalues do not reach the value of six. In case of $\Delta \theta=180^{\circ}$, the corresponding sum of eigenvalues tends toward the value of six, indicating the availability of six independent communication channels from the perspective of MIMO. Thus, from the following results it can be inferred that MIMO capacity increase by increasing angle spread factor for Line of Sight (LOS) and Non-Line of Sight (NLOS) environment.

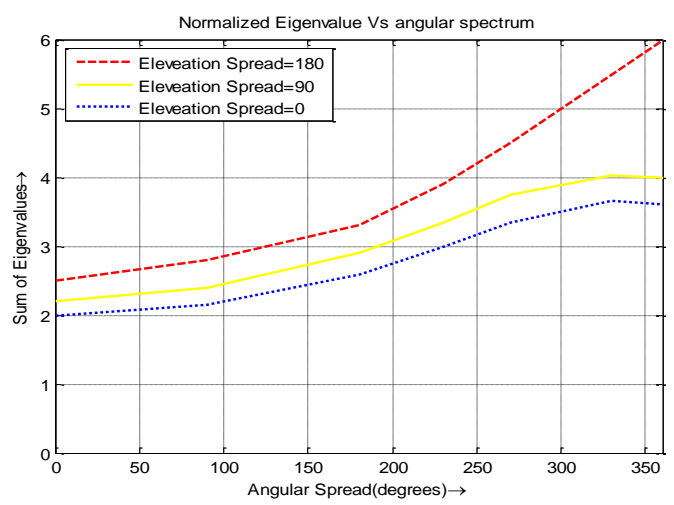

Fig. 4. Normalized sum of Eigenvalues of the correlation matrix vs. incident field angular spread

\subsection{Impact of element spacing $\lambda$ on channel capacity}

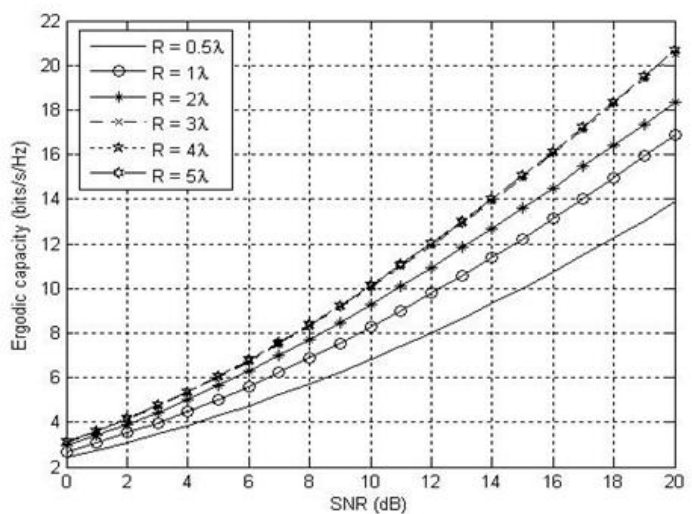

Figure 5 shows graph between ergodic channel capacities vs. SNR as a function of antenna spacing. The result is plotted for different values of antenna spacing i.e. $1 \lambda, 2 \lambda, 3 \lambda, 4 \lambda, 5 \lambda$. The figure clearly depicts that the channel capacity increases with an increase in element spacing. It also shows that while increasing the antenna spacing more than $3 \lambda$ has no effect on improving the capacity.

\section{CONCLUSION}

This paper deliberates the performance analysis of MIMO channel with polarization diversity to increase the channel capacity. MIMO systems implementing polarization diversity provides a considerable improvement in the overall channel capacity. The results depict that MIMO system is best suited for accessing all six electromagnetic field polarizations and also helps in mitigating the problem of correlation between the polarized field components at the receiver end. The study further investigates that capacity can be increased by increasing the element spacing or number of transmitting and receiving antennas. Moreover, the increase in angular spread can make sure the availability of all six independent channels integrating a MIMO system. Therefore, it is expected that this analysis will give intuition into the physical behavior prompting increased communication channels based upon electromagnetic field polarization. Thus, the proper implementation of such diverse MIMO systems is essential to meet the ubiquitous user demands of the future wireless generations.

\section{ACKNOWLEDGMENTS}

I owe a special debt of gratitude to my Professor Mr. Ashish Sharma Department of Computer Science and Engineering, Maharaja Agrasen Institute of Technology, Delhi for his constant dedication and support throughout the research. Without his thorough guidance and unique ideas I would not have been able to complete the research. I thank him for his sincerity, perseverance and constant motivation. 


\section{REFERENCES}

[1] Mujahid,U., Mudassir Mukhtar,J.A., Rehman,A., Abbas, M. \& Shahid,U. "Spectral Estimation for Smart Antenna System", $20133^{\text {rd }}$ International Conference on Computer, Control and Communication (IC4), pp. 1-5, 25-26 Sep. 2013.

[2] Langton, C., Sklar, B. "Tutorial 27 - Finding MIMO," Tutorials on Digital Communications Engineering, pp. 14, Oct. 2011.

[3] M. R. Andrews, P. P. Mitra, and R. deCarvalho, "Tripling the capacity of wireless communications using electromagnetic polarization," Nature, vol. 409, pp. 316318, Jan. 2001

[4] Dong, L., Choo, H., Heath, R. W., Ling, H., "Simulation of MIMO Channel Capacity with Antenna Polarization Diversity," IEEE Transactions on Wireless Communications, vol. 4, no. 4, pp. 1869 - 1873, Sep. 2005.

[5] Nandhini, R., Z. Yasmin, J., "Performance Enhancement of land Mobile Satellite System," International Journal of Emerging Technology and Advanced Engineering, vol. 3, no. 1, pp. 102-106, Jan. 2013.

[6] Sadiku, M. N. O., "Elements of Electromagnetics", 6th ed, McGraw Hill, New York, 2001.

[7] Svantesson, T., jensen, M. A., Wallace, J. W., "Analysis of Electromagnetic Field Polarizations in Multiantenna System," IEEE Transactions on Wireless Communications, vol. 2, no. 2, pp. 641-646, Mar. 2004.

[8] Gershman, A. B., Sidiropoulos, N. D., "Space-Time Processing for MIMO Communications," England: Jogn Wiley \& Sons Ltd, 2005.

[9] Saunders, Simon R., Aragon-Zavala, A., "Antennas and Propagation for Wireless Communication System," Second Edition, England: John Wiley \& Sons Ltd., 2007.

[10] Winters, J. H., "On the capacity of radio communication systems with diversity in a rayleigh fading environment,"
IEEE Journal on Selected Areas in Communications, vol. 5, no. 5, pp. 871-878, June 2003.

[11] Foschini, G. J., and Gans, M. J., "On limits of wireless communications in a fading environment when using multiple antennas," Wireless Personal Communications, vol. 6, pp. 311-335, Mar. 1998.

[12] Raleigh, G. G., and Cioffi, J. M., "Spatio-temporal coding for wireless communication," IEEE Transactions on Communications., vol. 46, pp. 357-366, Mar. 1998.

[13] T. Duman, M., Ghrayeb, Ali, "Coding For MIMO Communication Systems," England: John Wiley \& Sons Ltd., 2007.

[14] Hamalainen, J., Wichman, R., Nuutinen, J.-P., Ylitalo, J., "Analysis and Measurement for Indoor Polarization MIMO in $5.25 \mathrm{GHz}$ Band," IEEE $61^{\text {st }}$ Vehicular Technology Conference, vol. 1, pp. 252 - 256, 2005.

[15] Aziz, A., Rahim, M. K. A., Qadir M. F. A., Suaidi, M. K., "The Investigation of Polarization Diversity in MIMO System at $2.4 \mathrm{GHz}$," Journal of Telecommunication, Electronic and Computer Engineering, vol. 3, no, 2, pp. 47-54, Dec. 2011.

[16] Rappaport, Theodore S., "Wireless communications (principle and Practice)," Second Edition, Prentice Hall of India, 2007

[17] Proakis, J. G., "Digital Communication', Fourth Edition, McGraw-Hill Higher Education, 2001.

[18] D. Gesbert, H. Bolcskei, D. A. Gore, and A. Paulraj, "Outdoor MIMO wireless channels: model and performance prediction," IEEE Transactions on Communications, vol. 50, no. 12, pp. 1926-1934, Dec. 2002.

[19] J. W. Wallace and M. A. Jensen, "Modeling the indoor MIMO wirelesschannel," IEEE Transactions on Antennas Propagation ,vol. 50, no. 5, pp. 591-599, May 2002. 